\title{
COVID-19 vaccination and breast cancer surgery timing
}

\author{
Gary Ko ${ }^{1}(1) \cdot$ Susy Hota ${ }^{1} \cdot$ Tulin D. Cil $^{1}$
}

Received: 4 June 2021 / Accepted: 12 June 2021 / Published online: 22 June 2021

(c) The Author(s), under exclusive licence to Springer Science+Business Media, LLC, part of Springer Nature 2021

\begin{abstract}
There have been recent reports in the breast imaging literature of unilateral axillary lymphadenopathy following COVID19 vaccination. It is unclear whether the reactive lymphadenopathy may impact the sentinel lymph node biopsy procedure. In this article, we provide guidelines regarding the timing of the COVID-19 vaccine and breast cancer surgery which were formulated after a review of the available literature and in consultation with infectious disease specialists.
\end{abstract}

Keywords Breast Cancer · Covid-19 · Vaccine - Lymphadenopathy

There have been recent reports in the breast imaging literature of unilateral axillary lymphadenopathy following COVID-19 vaccination. It has been estimated to occur in $11.6 \%$ of people after receiving their first dose and $16 \%$ of people after receiving their second dose of the Moderna COVID-19 vaccine [1]. This has prompted a proposal for breast screening to occur either before a patient receives the COVID-19 vaccine or 4-6 weeks after, in order to avoid false positives on imaging. Physicians should inquire about recent COVID-19 vaccination for patients who have unilateral axillary lymphadenopathy. In patients who have unilateral axillary lymphadenopathy on imaging and a history of receiving a COVID-19 vaccine in the ipsilateral arm within 4 weeks, short term follow-up imaging in 4-12 weeks after the second vaccine dose should be considered instead of excision. If the lymphadenopathy persists on follow-up imaging, lymph node sampling (e.g. FNA or core biopsy) should be performed to exclude malignancy [2].

Most breast cancer surgery involves the retrieval of sentinel lymph nodes. It is unclear whether the reactive lymphadenopathy may affect the accuracy of the sentinel lymph node biopsy procedure. Furthermore, side effects secondary to the vaccine (such as low-grade fever) may be similiar to usual post-operative symptoms. The key is to minimize risk of confusing symptoms that may lead to over-diagnosis of surgical site infections (e.g. skin rashes, enlarged lymph nodes)

Gary Ko

gary.ko@mail.utoronto.ca

1 University Health Network, 700 University Ave, 6th floor, Toronto, ON M5G 1X6, Canada when they are actually due to lingering vaccine-related side effects.

We have not found any guidelines regarding the timing of the COVID-19 vaccine and breast cancer surgery. As such, our breast surgical oncology service has formulated the following recommendations after a review of the available literature [3] and in consultation with our infectious disease specialists at the University Health Network in Toronto, Ontario, Canada.

1) We recommend scheduling the COVID-19 vaccine at least one week before surgery so symptoms such as fever can be correctly attributed to side effects from the vaccine rather than surgery.

2) Since the COVID-19 vaccines can cause lymphadenopathy, we recommend the vaccine be administered on the OPPOSITE arm to the affected breast, if possible. The anterolateral thigh can be also be considered as an injection site.

3) Post-operative antibiotics (e.g. commonly prescribed after breast reconstructive surgery) should not interfere with administration of the COVID-19 vaccine.

4) Vaccination can also occur once patients are recovered, one to two weeks after breast surgery. This timing can be re-assessed on an individual basis and is dependent on the clinical status of the patient.

Ultimately, the risks of delaying a COVID-19 vaccine for surgical therapy needs to be balanced with the risks of reactive lymphadenopathy and possibly masking side effects of surgery. Patients should make an informed decision after 
discussing with their physicians about the timing of COVID19 vaccine with their breast cancer treatment. ${ }^{\mathrm{TM}}$

Authors' contributions GK and TC were involved in conceptualization and writing of the manuscript. SH provided expert opinion and was involved in editing the manuscript. All authors have read and approved the final manuscript.

Funding None.

Availability of data and material Not applicable.

Code availability Not applicable.

\section{Declarations}

Conflicts of interests The authors declare that they have no competing interests.

Ethics approval Not applicable.
Consent to participate Not applicable.

Consent for publication Not applicable.

\section{References}

1. Local Reactions, Systemic Reactions, Adverse Events, and Serious Adverse Events: Moderna COVID-19 Vaccine I CDC. https:// www.cdc.gov/vaccines/covid-19/info-by-product/moderna/react ogenicity.html. Accessed 22 Mar 2021

2. Seely JM, Barry MH (2021) The Canadian society of breast imaging/ canadian association of radiologists' recommendations for the management of axillary adenopathy in patients with recent COVID-19 vaccination. Can Assoc Radiol J. https://doi.org/10. $1177 / 0846537121998949$

3. Vaccinated patients guidance. In: Royal College of Surgeons. https://www.rcseng.ac.uk/coronavirus/vaccinated-patients-guida nce/. Accessed 15 Mar 2021

Publisher's Note Springer Nature remains neutral with regard to jurisdictional claims in published maps and institutional affiliations. 